G. Gallavotti and H. P. McKean

Nagoya Math. J.

Vol. 47 (1972), 1-14

\title{
BOUNDARY CONDITIONS FOR THE HEAT EQUATION IN A SEVERAL-DIMENSIONAL REGION*
}

\author{
G. GALLAVOTTI AND H. P. MCKEAN
}

\begin{abstract}
The heat equation $\partial p / \partial t=\Delta p / 2$ is to be solved in a severaldimensional region $D$ with $\partial p=k p+j \Delta p / 2$ on the boundary $B$ of $D$. The elementary solution (Green's function) is interpreted as the transition density of an associated Brownian motion. The latter is built up pathwise from the free Brownian motion by simple geometric and probabilistic transformations.
\end{abstract}

1. Introduction. The heat equation $\partial p / \partial t=\Delta p / 2$ is to be solved in a several-dimensional region $D$ with $\partial p=k p+j \Delta p / 2$ on the boundary $B$ of $D$. The letter $\partial$ stands for differentiation in the inward-pointing direction perpendicular to $B$, and $k$ and $j$ are non-negative functions of class $C(B)$. The elementary solution (Green's function) of this problem is interpreted as the transition density of an associated Brownian motion. The latter is built up pathwise from the free Brownian motion by simple geometric and probabilistic transformations. The "reflecting" Brownian motion $(k=j=0)$ is constructed by means of a covering surface (method of images) in section 3 after the necessary facts about the 1-dimensional Brownian motion have been explained in section 2. The "elastic" Brownian motion $(j=0)$ is constructed in articles 4.1-3, the "sticky" Brownian motion $(k=0)$ in article 4.4, and the Brownian motion for general $k$ and $\mathrm{j}$ article 4.5. The presentation is a bit skimpy in places, the purpose being to explain the idea in a (hopefully) self-contained way without getting bogged down in technical details: for this reason, the proofs deal only with polygonal regions $D$ bounded by a finite number of flat faces of codimension 1, such as, in 3 dimensions, triangles, half-

Received October 11, 1971.

* The work of the second author was supported in part by the Office of Naval Research, Contract N00014-67-A-0467-0014. Reproduction in whole or in part is permitted for any purpose of the United States Government. 
planes, pentagons, and the like. An exhaustive account of the possible Brownian motions on a half-line may be found in [3]. The connection with the appropriate quadratic forms

$$
D[f]+B[f]=\int_{D}|\operatorname{grad} f|^{2}+\text { a quadratic form on } B
$$

will be the subject of a second paper; see also [2] for such explanations in a more general setting.

\section{Simple Brownian Motions.}

2.1. One-Dimensional Free Brownian Motion. The free 1-dimensional Brownian motion $\mathfrak{x}^{\circ}$ starting at $x \in R^{1}$ is defined by extending the probabilities

$$
\begin{gathered}
P\left[\bigcap_{i \leq n}\left(a_{i} \leq \mathfrak{x}^{\circ}\left(t_{i}\right)<b_{i}\right)\right]=\int_{a_{1}}^{b_{1}} \ldots \int_{a_{n}}^{b_{n}} \frac{e^{-\left(x_{1}-x\right) 2 / 2 t_{1}}}{\sqrt{2 \pi t_{1}}} \frac{e^{-\left(x_{2}-x_{1}\right) 2 / 2\left(t_{2}-t_{1}\right)}}{\sqrt{2 \pi\left(t_{2}-t_{1}\right)}} \\
\cdots \frac{e^{-\left(x_{n}-x_{n-1}\right) 2 / 2\left(t_{n}-t_{n-1}\right)}}{\sqrt{2 \pi\left(t_{n}-t_{n-1}\right)}} d^{n} x
\end{gathered}
$$

from "cylinder sets" $\cap\left(a_{i} \leq \mathfrak{x}^{\circ}\left(t_{1}\right)<b_{i}\right)$ to the natural class of Borel sets of the space of continuous paths $\mathfrak{x}^{\circ}:[0, \infty) \rightarrow R^{1}$; see for example [3: 12-22]. The basic ingredient of this construction is the Gauss kernel

$$
p^{\circ}(t, x, y)=(2 \pi t)^{-1 / 2} \exp \left[-(x-y)^{2} / 2 t\right]
$$

in its dual role as a) the free Brownian transition density and b) the elementary solution (Green function) of $\partial p / \partial t=(1 / 2) \partial^{2} p / \partial x^{2}$. The chief feature of the motion is that the Brownian traveller has no memory. To express this fact in a precise way, let $T$ be a constant time or even a passage time such as $T=\min \left(t: \mathfrak{x}^{\circ}(t)=1\right)$. Then, conditional on the past $\mathfrak{x}^{\circ}(s): s \leq T$, the future $\mathfrak{x}^{+}(t)=\mathfrak{x}^{\circ}(t+T): t \geq 0$ is a free Brownian motion starting at $y=\mathfrak{x}^{\circ}(T)$. This may be stated more informally by saying that the free Brownian motion begins afresh at such times $T$. A simple proof may be found in [6:10-11].

2.2 Brownian Differentials and Integrals. The free Brownian motion $\mathfrak{r}^{\circ}$ has associated with it a nice differential and integral calculus. The differential of a smooth function $f$ composed with $\mathfrak{c}^{\circ}(t)$ begins with $f^{\prime}\left(x^{\circ}\right) d x^{\circ}$, as you would expect, but you have to keep the second 
term $(1 / 2) f^{\prime \prime}\left(\mathfrak{d}^{\circ}\right)\left(d \mathfrak{r}^{\circ}\right)^{2}$ of the power series and interpret $\left(d \mathfrak{x}^{\circ}\right)^{2}$ as $d t$. This rule stems from the fact that

$$
\dot{P}\left[\lim _{n \uparrow \infty} \sum_{k 2-n}\left|\mathfrak{x}^{\circ}\left(\frac{k}{2^{n}}\right)-\mathfrak{x}^{\circ}\left(\frac{k-1}{2^{n}}\right)\right|^{2}=t \text { for every } t \geq 0\right]=1 .
$$

The differential formula is meant to signify that

$$
f\left[\mathfrak{x}^{\circ}(t)\right]-f\left[\mathfrak{x}^{\circ}(0)\right]=\int_{0}^{t} f^{\prime}\left[\mathfrak{x}^{\circ}(s)\right] d \mathfrak{x}^{\circ}(s)+\frac{1}{2} \int_{0}^{t} f^{\prime \prime}\left[\mathfrak{x}^{\circ}(s)\right] d s .
$$

Warning: For the integral formula to be correct, the differentials in the Brownian integral must stick out into the future, as in

$$
\int_{0}^{t} f^{\prime}\left(\mathfrak{c}^{\circ}\right) d \mathfrak{\complement}^{\circ}=\lim _{n \uparrow 0} \sum_{k 2-n} f^{\prime}\left[\mathfrak{c}^{\circ}\left(\frac{k-1}{2^{n}}\right)\right]\left[\mathfrak{x}^{\circ}\left(\frac{k}{2^{n}}\right)-\mathfrak{x}^{\circ}\left(\frac{k-1}{2^{n}}\right)\right] .
$$

This has the additional advantage that $E\left(\int f^{\prime} d \mathfrak{x}^{\circ}\right)=0$. A complete account of Brownian differentials and integrals may be found in [6].

2.3 Brownian Local Time. An important ingredient of the explanations below is the "local time" of P. Lévy [5]:

$$
\mathfrak{t}^{\circ}(t)=\lim _{\varepsilon \downarrow 0}(2 \varepsilon)^{-1} \text { measure }\left(s \leq t:\left|\mathfrak{x}^{\circ}(s)\right|<\varepsilon\right) .
$$

To make it plausible that such an object exists, let $f$ be the product of $(2 \varepsilon)^{-1}$ and the indicator function of $|x|<\varepsilon$, and let $I$ be the integral $I: f \rightarrow \int_{-\infty}^{x} f$. By the Brownian differential recipe of article 2.2,

$$
\begin{aligned}
& (2 \varepsilon)^{-1} \text { measure }\left(s \leq t:\left|\mathfrak{x}^{\circ}(s)\right|<\varepsilon\right) \\
& =\int_{0}^{t} f\left(\mathfrak{\complement}^{\circ}\right) d s=\int_{0}^{t}\left(I^{2} f\right)^{\prime \prime}\left(\mathfrak{c}^{\circ}\right) d s=2 I^{2} f \mathfrak{E}_{\mathfrak{E}^{\circ}(0)}^{\mathfrak{r}^{\circ}(0)}-2 \int_{0}^{t} I f\left(\mathfrak{d}^{\circ}\right) d \mathfrak{d}^{\circ},
\end{aligned}
$$

and as $\varepsilon \downarrow 0$, it is pretty easy to see that this approaches

$$
2 \max \left[\mathfrak{x}^{\circ}(t), 0\right]-2 \max \left[\mathfrak{x}^{\circ}(0), 0\right]-2 \int_{0}^{t} 1\left(\mathfrak{x}^{\circ}\right) d \mathfrak{x}^{\circ},
$$

in which 1 stands for the indicator function of the half-line $x \geq 0$. This proves that $\mathfrak{t}^{\circ}$ exists.

2.4 Reflecting Brownian Motion. The next thing you need is the "reflecting" Brownian motion $\mathfrak{x}^{+}=\left|\mathfrak{x}^{\circ}\right| \mathfrak{r}^{+}$has no memory, as can 
be seen from the fact that $-\mathfrak{x}^{\circ}$ is also a free Brownian motion, and it is easy to check that the associated transition density

$$
p^{+}(t, x, y)=(2 \pi t)^{-1 / 2}\left[e^{-(x-y) 2 / 2 t}+e^{-(x+y)^{2} / 2 t}\right]
$$

is the Green function of $\partial p / \partial t=(1 / 2) \partial^{2} p / \partial x^{2}$ for $x>0$, subject to $\partial p=0$ at $x=0$. P. Lévy [5] discovered a second way of constructing the reflecting Brownian motion. The existence of the local time of article 2.3 will be confirmed thereby at no extra cost. Bring in the maximum function

$$
\mathrm{t}^{-}(t)= \begin{cases}0 & \text { if } t<T=\min \left(s: \mathfrak{x}^{\circ}(s)=0\right) \\ \max _{T \leq s \leq t} \mathfrak{x}^{\circ}(s) & \text { if } t \geq T\end{cases}
$$

for free Brownian paths starting in $[0, \infty)$. Then

$$
\mathfrak{x}^{-}= \begin{cases}\mathfrak{x}^{\circ} & \text { if } t<T \\ \mathfrak{t}^{-}-\mathfrak{x}^{0} & \text { if } t \geq T\end{cases}
$$

is a reflecting Brownian motion (that is to say, it is identical in law to $\left.\mathfrak{x}^{+}\right)$, and $\mathfrak{t}^{-}$is its local time:

$$
\mathfrak{t}^{-}(t)=\lim _{s \downarrow 0}(2 \varepsilon)^{-1} \text { measure }\left(s \leq t: \mathfrak{x}^{-}(s)<\varepsilon\right) .
$$

The nice thing is that the statement can be turned around: the customary reflecting Brownian motion $\mathfrak{x}^{+}=\left|\mathfrak{x}^{\circ}\right|$ has a local time

$$
\mathfrak{t}^{+}(t)=\lim _{\varepsilon \downarrow 0}(2 \varepsilon)^{-1} \text { measure }\left(s \leq t: \mathfrak{x}^{+}(s)<\varepsilon\right),
$$

and just as

$$
\mathfrak{x}^{-}-\mathfrak{t}^{-}=\left\{\begin{aligned}
\mathfrak{x}^{\circ} & \text { if } t<T \\
-\mathfrak{x}^{\circ} & \text { if } t \geq T
\end{aligned}\right.
$$

is a free Brownian motion, so also is $\mathfrak{x}^{+}-\mathfrak{t}^{+}$, that is to say, $\mathfrak{x}^{+}=\mathfrak{t}^{+}+a$ free Brownian motion.

Warning: The free Brownian motion $\mathfrak{x}^{+}-t^{+}$is not pathwise the same as the free Brownian motion $\mathfrak{x}^{\circ}$ figuring in $\mathfrak{x}^{+}=\left|\mathfrak{x}^{\circ}\right| \cdot$ The statement is merely that they have the same statistics. The actual relationship between them is very complicated but fortunately it is not necessary to know it.

The proofs of the above can be found in [4:40-42]. 
2.5 Reflecting Brownian Motion on an Interval. The reflecting motion on an interval $(0 \leq x \leq 1$, say) corresponding to $\partial p / \partial t=$ $(1 / 2) \partial^{2} p / \partial x^{2}$ with $\partial p=0$ at both ends may be constructed from the free Brownian motion by a simple folding recipe (method of images), elaborating the single fold $x \rightarrow|x|$. To do this, think of the line as hinged at each integral point and fold it up into a unit interval as in Figure 1. This projection maps the free Brownian motion $\mathfrak{x}^{\circ}$ into the reflecting Brownian motion on $0 \leq x \leq 1$. The corresponding transition density is

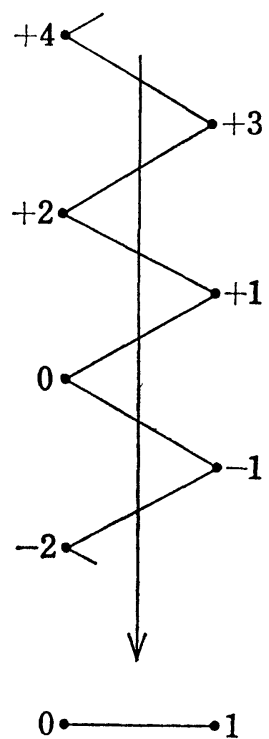

Fig. 1

$$
p^{+}(t, x, y)=\sum_{n \text { even }}(2 \pi t)^{-1 / 2}\left[e^{-(x-y+n) 2 / 2 t}+e^{-(x+y+n) 2 / 2 t}\right]
$$

The reader is invited to check (by pure thought) that the folded motion has no memory and that $p^{+}$really is the Green function of the stated problem.

2.6 Free Brownian Motion in Several Dimensions. The free $d$-dimensional Brownian motion $\mathfrak{c}^{\circ}$ is just the joint motion of $d$ independent free 1-dimensional Brownian particles. The transition density is now

$$
p^{\circ}(t, x, y)=(2 \pi t)^{-d / 2} \exp \left[-|x-y|^{2} / 2 t\right]
$$


which is likewise the Green function of $\partial p / \partial t=\Delta p / 2$. The lack of memory is as before, and the differential and integral calculus of article 2.2 is easily adapted. The only new point is that the old rule $\left(d x^{\circ}\right)^{2}=d t$ is supplemented by $d \mathfrak{x}_{i}^{\circ} d \mathfrak{x}_{j}^{\circ}=0$ if $i \neq j$.

3 Reflecting Brownian Motion in a Polygonal Region. The purpose of this section is to construct the reflecting Brownian motion in a severaldimensional polygonal region $D$ by a "folding recipe" similar to that of article 2.5. The adjective "polygonal" means that the boundary $B$ of $D$ is the sum of a finite number of flat pieces of codimension 1 . $D$ may bounded or not, and the dimension $d$ can be anything, but for simplicity and ease of illustration $d=2$ is fixed from now on. $B$ is now the sum open edges plus a finite number of corners, designated by $C$.

3.1 The Half-Plane. The simplest case is that of a half-plane $D=\left(x_{1}>0,-\infty<x_{2}<\infty\right)$, say. The corresponding reflecting Brownian motion is $\mathfrak{x}^{+}=\left(\mathfrak{x}_{1}^{+}, \mathfrak{x}_{2}^{\circ}\right)$ in which $\mathfrak{x}_{1}^{+}=\left|\mathfrak{x}_{1}^{\circ}\right|$ is a 1-dimensional reflecting Brownian motion and $\mathfrak{x}_{2}^{\circ}$ is an independent free 1-dimensional Brownian motion. To put the matter more geometrically, $\mathfrak{x}^{+}$is made from the free 2-dimensional Brownian motion $\mathfrak{x}^{\circ}=\left(\mathfrak{x}_{1}^{\circ}, \mathfrak{x}_{2}^{\circ}\right)$ by folding the plane along the line $B:\left(x_{1}=0\right)$. A second description $\mathfrak{x}^{+}=\mathfrak{x}^{\circ}+\mathfrak{t}$ is obtained by expressing $\mathfrak{d}_{1}^{+}$as $\mathfrak{x}_{1}^{\circ}+\mathrm{t}_{1}^{+}$with a new free 1-dimensional Brownian motion $\mathfrak{x}_{1}^{\circ}$. The new plane motion $\mathfrak{x}^{\circ}=\left(\mathfrak{x}_{1}^{\circ}, \mathfrak{x}_{2}^{\circ}\right)$ is a free 2-dimensional Brownian motion, and the (directed) local time $t$ is the product of $t_{1}^{+}$and the inward-pointing unit direction perpendicular to $B$. The fact that $D$ is a special half-plane is of no account: any rigid motion (rotation or translation) of the free Brownian motion $\mathfrak{x}^{\circ}$ is likewise a free Brownian motion, so the above applies to any half-plane you like.

3.2 A Covering Surface. The folding recipe of article 3.1 may be adapted to any polygonal region $D$ by means of a simple covering surface closely related to Kelvin's "method of images"; see also [7: 7982]. Think, for simplicity, of a triangle $D$ with sides 123 and label it with a 0 as in Figure 2. The covering is obtained by repeated reflection of $D$ across its sides: each new reflection produces a copy of the "fundamental domain" $D$ labelled by a string of integers listing (from left to right) the successive sides employed for reflection, and these images are declared to be all different, so that you get an infinite-sheeted covering 


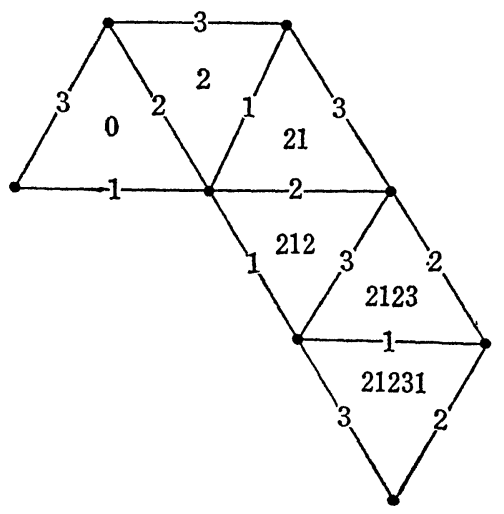

Fig. 2

$K$ of $D$ with one sheet to each possible string of reflections, as in the figure. The situation may be clarified by thinking of the case of an equilateral triangle. $K$ is not the conventional universal covering depicted in Figure 3: for example, the image 23232 is identified with the image 3 in the universal cover, but they are distinguished in $K$. The covering $K$ is provided with a self-evident projection onto $D+B$ and the "folded" motion $\mathfrak{c}^{+}$on $D+B-C$ is simply the projection of a free Brownian motion $\mathfrak{x}^{\circ}$ on the open manifold $M=K$ - (images of corners) as explained in the next article. The reader will compare this recipe with that of article 2.5.

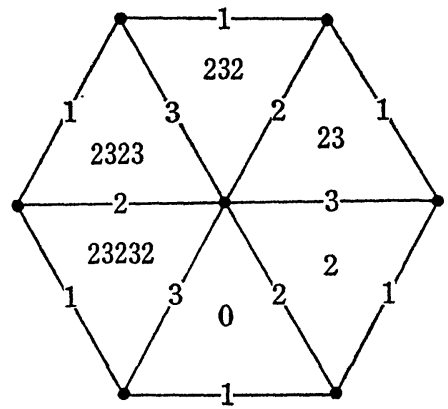

Fig. 3

3.3 Free Brownian Motion on $M$. The free Brownian motion $\mathfrak{x}^{\circ}$ on $M$ can be pictured as follows. Pick a point of $M$, start a free 2-dimensional Brownian motion there, and let it run. The images of corners are countable, so the path will never hit one; instead, it will cross from one copy of $D$ into another via images of the open edges of $B$, and 
so continue forever. $M$ carries a self-evident area element, also $\Delta$ makes sense, and it is easy to see that the transition density $p^{\circ}=p^{\circ}(t, x, y)$ of the above free Brownian motion is of class $C^{\infty}\left[(0, \infty) \times M^{2}\right]$ and is the Green function of $\partial p / \partial t=\Delta p / 2$ on $M$. To prove this, let $f$ be a compact function of class $C^{\infty}[(0, \infty) \times M]$ and compute the differential of $f\left[t, \mathfrak{x}^{\circ}(t)\right]$ according to the rules laid down in articles 2.2 and 2.6 :

$$
d f\left[t, \mathfrak{x}^{\circ}(t)\right]=\frac{\partial f}{\partial t} d t+\operatorname{grad} f d \mathfrak{x}^{\circ}+\frac{\Delta f}{2} d t .
$$

Now integrate back from 0 to $\infty$ and take the expectation of both sides for paths starting at a fixed point $x \in M$. This gives

$$
\begin{aligned}
0 & =\left.E f\left[t, \mathfrak{x}^{\circ}(t)\right]\right|_{0} ^{\infty}=E \int_{0}^{\infty}\left(\frac{\partial}{\partial t}+\frac{\Delta}{2}\right) f\left[t, \mathfrak{x}^{\circ}(t)\right] d t \\
& =\int_{0}^{\infty} d t \int_{M} p^{\circ}(t, x, y)\left(\frac{\partial}{\partial t}-\frac{\Delta}{2}\right) f(t, y) d y
\end{aligned}
$$

in which $d y$ is the element of area on $M$ and $p^{\circ}(t, x, y)$ is the (formal) transition density for $\mathfrak{x}^{\circ}$. But now a celebrated lemma of $\mathrm{H}$. Weyl states that such a formal density is actually of class $C^{\infty}[(0, \infty) \times M]$ so that you may integrate by parts to obtain $\partial p / \partial t=\Delta p / 2$ in the customary sense; see for example [1] or [6:85-90]. The fact that $p^{\circ}$ is smooth on $(0, \infty) \times M^{2}$ now follows from its self-evident symmetry in $x$ and $y$.

3.4 Identification of $\mathfrak{x}^{+}$as Reflecting Brownian Motion. To say that $\mathfrak{x}^{+}$is the reflecting Brownian motion on $D+B-C$ means that its transition density $p^{+}=p^{+}(t, x, y)$ is the Green function with pole at $x=y$ of $\partial p / \partial t=\Delta p / 2$ subject to $\partial p=0$ on $B-C$. Pick $x$ and $y$ from $D+B-C$. Then

$$
p^{+}(t \cdot x \cdot y)=\sum p^{\circ}\left(t, x, y^{\prime}\right)
$$

in which $p^{\circ}$ is the free Brownian transition density and $y^{\prime}$ runs through the images of $y$ on the covering surface $K$. Because $p^{\circ}$ solves $\partial p / \partial t=$ $\Delta p / 2$ on $M$, a simple application of Weyl's lemma proves that $p^{+}$is of class $C^{\infty}\left[(0, \infty) \times(D+B-C)^{2}\right]$ and solves $\partial p / \partial t=\Delta p / 2$ in $D$; in fact, it shows that $p^{+}$is smooth across $B-C$, and since the sum for $p^{+}$is even across $B-C$, you see that $\partial p^{+}=0$ on $B-C$. The fact that the pole is located at $x=y$ is self-evident, and the only moot point is whether $p^{+}$is the only such function. 
3.5 Local Times. To settle this point, it is advantageous to express $\mathfrak{x}^{+}$as the sum $\mathfrak{x}^{\circ}+t$ of a (new) free 2-dimensional Brownian motion $\mathfrak{x}^{\circ}$ and a (directed) local time $t$, as for the half-plane of article 3.1. The differential $d t$ vanishes unless $\mathfrak{x}^{+} \in B$, in which case it points in the inward direction perpendicular to the side of $B$ on which $\mathfrak{x}^{+}$finds itself. The (absolute) local time spent on $B$ is declared to be

$$
|t|(t)=\int_{0}^{t}|d t|=\lim _{\varepsilon \downarrow 0}(2 \varepsilon)^{-1} \text { measure }\left(s \leq t: \mathfrak{x}^{+}(s) \text { at distance }<\varepsilon \text { from } B\right) .
$$

3.6 Uniqueness. The fact that $p^{+}$is the only Green function of $\partial p / \partial t=\Delta p / 2$ with $\partial p=0$ on $B-C$ is now proved by checking that $\int_{D} p^{+} f d y$ is the only bounded solution of
a) $u \in C^{\infty}[(0, \infty) \times(D+B-C)]$
b) $\partial u / \partial t=\Delta u / 2 \quad$ in $(0, \infty) \times D$
c) $\partial u=0 \quad$ on $(0, \infty) \times(B-C)$
d) $u(0+, \cdot)=f \quad$ in $D+B-C$.

Evidently, it is enough to check that if $f=0$ in d), then $u=0$. Fix $0<T$. By a), b), c), and the rules for Brownian differentials,

$$
\begin{aligned}
d u\left[T-t, \mathfrak{x}^{+}(t)\right] & =-\frac{\partial u}{\partial t} d t+\operatorname{grad} u d \mathfrak{c}^{+}+\frac{\Delta u}{2} d t \\
& =\left(-\frac{\partial}{\partial t}+\frac{\Delta}{2}\right) u d t+\operatorname{grad} u d \mathfrak{c}^{\circ}+\partial u d|\mathrm{t}| \\
& =\operatorname{grad} \mathrm{u} d \mathfrak{c}^{\circ},
\end{aligned}
$$

so by d) with $f=0$,

$$
-u\left[T, \mathfrak{x}^{+}(0)\right]=\int_{0}^{T} \operatorname{grad} u\left[T-t, \mathfrak{x}^{+}(t)\right] d \mathfrak{x}^{\circ} .
$$

But also, for fixed $\mathfrak{x}^{+}(0) \in D+B-C$,

$$
\begin{aligned}
E \int_{0}^{T}|\operatorname{grad} u|^{2} d t & =\lim _{R \uparrow T} E \int_{0}^{R}|\operatorname{grad} u|^{2} d t \\
& =\lim E\left|\int_{0}^{R} \operatorname{grad} u d \mathfrak{c}^{\circ}\right|^{2} \\
& =\lim E\left|u\left[T-R, \mathfrak{c}^{+}(R)\right]-u\left[T, \mathfrak{x}^{+}(0)\right]\right|^{2}<\infty,
\end{aligned}
$$


and this makes $E \int_{0}^{T} \operatorname{grad} u d \mathfrak{r}^{\circ}=0[6: 25]$, i.e., $u=0$, as advertised.

4 General Barriers. Now you are in a position to understand the "barrier" $\partial p=k p+j \Delta p / 2$ for general $k$ and $j$. The "elastic" barrier $(j=0)$ is treated in articles $4.1-3$, the "sticky" barrier $(k=0)$ in article 4.4 , and the general barrier in article 4.5 .

4.1 Elastic Barriers. The idea is that the elastic Brownian motion corresponding to $\partial p=k p$ on $B-C$ is just the reflecting Brownian motion $\mathfrak{x}^{+}=\mathfrak{x}^{\circ}+\mathfrak{t}$ killed at rate proportional to the amount time the latter spends on $B$. Because measure $\left(t: \mathfrak{x}^{+} \in B\right)=0$, you cannot reckon this by the standard clock $t$. As a substitute, the (absolute) local time $|t|$ suggests itself, and it can be argued that the chance of getting killed in time $d t$ having survived up to time $t$ should be something like $k\left(\mathfrak{x}^{+}\right) d|\mathrm{t}|$. The change of not getting killed before time $t$ would then be the product of $1-k\left(\mathfrak{x}^{+}\right) d|\mathrm{t}|$ for $s \leq t$, alias $e^{-\mathfrak{r}}$ with $\mathfrak{t}=\int_{0}^{t} k\left(\mathfrak{x}^{+}\right) d|t|$, so the final form of the proposal would be that if

$$
\mathfrak{d}^{\infty}= \begin{cases}\mathfrak{x}^{+} & \text {if } t<T \\ \infty & \text { if } t \geq T,\end{cases}
$$

and if the "killing time" $T$ is distributed according to the conditional law $P\left(T>t \mid \mathfrak{x}^{+}\right)=e^{-\mathfrak{t}}$, then the associated transition density $p^{\infty}$ will be the Green function of $\partial p / \partial t=\Delta p / 2$ in $D$, subject to $\partial p=k p$ on $B-C$.

4.2 The Half-Line. To begin with the simplest case, let $D+B$ be the half-line $[0, \infty)$. Then $k$ is just a constant, and you have to check that the transition density $p^{\infty}$ defined by the rule

$$
\begin{aligned}
p^{\infty}(t, x, y) d y & =E\left[e^{-k t+}, \mathfrak{c}^{+}(t) \in d y\right]=P\left[\mathfrak{x}^{+}(t) \in d y, T>t\right] \\
& =P\left[\mathfrak{c}^{\infty}(t) \in d y\right]
\end{aligned}
$$

is the elementary solution of $\partial p / \partial t=(1 / 2) \partial^{2} p / \partial x^{2}$ with $\partial p=k p$ at $x=0$. This is easily verified by a direct computation. If $T_{0}$ is the free Brownian passage time $\min \left(t: \mathfrak{c}^{\circ}(t)=0\right)$, then

$$
P\left[T_{0} \in d t\right]=\left(2 \pi t^{3}\right)^{-1 / 2} x e^{-x^{2} / 2 t} d t
$$

for paths starting at $x>0$, while for paths starting at $x=0$, 


$$
P\left[\mathfrak{x}^{\circ}(t) \in d a, \mathfrak{t}^{-}(t) \in d b\right]= \begin{cases}0 & \text { if } a \geq b \\ \left(2 / \pi t^{3}\right)^{-1 / 2}(2 b-a) e^{-(2 b-a) 2 / 2 t} d a d b & \text { if } a<b .\end{cases}
$$

Therefore, by the recipe $\mathfrak{t}^{-}-\mathfrak{x}^{\circ}$ for the reflecting Brownian motion $\mathfrak{x}^{-}$ of article 2.4,

$$
\begin{aligned}
p^{\infty}(t, x, y)= & \frac{\partial}{\partial y} P\left[\mathfrak{x}^{\circ}(t) \leq y, T_{0}>t\right] \\
& +\frac{\partial}{\partial y} P\left[T_{0} \leq t, e^{-k \mathfrak{t}-(t)}, \mathfrak{t}^{-}(t)-\mathfrak{c}^{\circ}(t) \leq y\right] \\
= & (2 \pi t)^{-1 / 2}\left[e^{-(x-y) 2 / 2 t}-e^{-(x+y)^{2 / 2} t}\right] \\
& +\int_{0}^{t}\left(2 \pi s^{3}\right)^{-1 / 2} e^{-x^{2} / 2 s} d s \\
& \cdot \int_{0}^{\infty} e^{-k b}\left(2 / \pi(t-s)^{3}\right)^{1 / 2}(b+y) e^{-(b+y) 2 / 2(t-s)} d b \\
= & (2 \pi t)^{-1 / 2}\left[e^{-(x-y) 2 / 2 t}-e^{-(x+y) 2 / 2 t}\right] \\
& +e^{k(x+y)} \int_{x+y}^{\infty} e^{-k b}\left(2 / \pi t^{3}\right)^{1 / 2} b e^{-b^{2} / 2 t} d b
\end{aligned}
$$

and now it is easy to check by hand that $p^{\infty}$ does everything it should.

4.3 Polygonal Regions. The identification of $p^{\infty}$ for polygonal regions $D$ is not so cheap. Pick a compact function $f$ of class $C^{\infty}[(0, \infty)$ $\times(D+B-C)]$, compute the differential

$$
d e^{-\mathfrak{t}} f\left[t, x^{+}(t)\right]=e^{-\mathfrak{t}}\left[\left(\frac{\partial}{\partial t}+\frac{\Delta}{2}\right) f d t+\operatorname{grad} f d \mathfrak{x}^{\circ}+(\partial-k) f d|\mathfrak{t}|\right],
$$

and integrate back as in article 3.3 to obtain

$$
\begin{aligned}
0= & \left.E e^{-\mathfrak{f}} f\left(t \cdot \mathfrak{c}^{+}\right)\right|_{0} ^{\infty}=E \int_{0}^{\infty} d e^{-\mathfrak{t}} f\left(t, \mathfrak{\mathfrak { c }}^{+}\right) \\
= & E \int_{0}^{\infty} e^{-\mathfrak{f}}\left(\frac{\partial}{\partial t}+\frac{\Delta}{2}\right) f\left(t, \mathfrak{c}^{+}\right) d t \\
& +E \int_{0}^{\infty} e^{-\mathfrak{t}}(\partial-k) f\left(t, \mathfrak{c}^{+}\right) d|\mathfrak{t}|
\end{aligned}
$$

for fixed $x=\mathfrak{x}^{\infty}(0)=\mathfrak{x}^{+}(0) \in D+B-C$. The expectation in line 3 is easily converted into

$$
E \int_{0}^{T}\left(\frac{\partial}{\partial t}+\frac{\Delta}{2}\right) f\left(t, \mathfrak{r}^{\infty}\right) d t=\int_{0}^{\infty} d t \int_{D} p^{\infty}\left(\frac{\partial}{\partial t}+\frac{\Delta}{2}\right) f,
$$


while with a little extra effort the expectation in line 4 is seen to be the same as

$$
E \int_{0}^{T}(\partial-k) f\left(t, \mathfrak{x}^{\infty}\right) d|\mathrm{t}|=\frac{1}{2} \int_{0}^{\infty} d t \int_{B} p^{\infty}(\partial-k) f
$$

Therefore,

$$
0=\int_{0}^{\infty} d t \int_{D} p^{\infty}\left(\frac{\partial}{\partial t}+\frac{\Delta}{2}\right) f+\frac{1}{2} \int_{0}^{\infty} d t \int_{B} p^{\infty}(\partial-k) f,
$$

and a formal application of Green's formula to the first integral produces the (formula) identity

$$
\begin{aligned}
0= & \int_{0}^{\infty} d t \int f\left(-\frac{\partial}{\partial t}+\frac{\Delta}{2}\right) p^{\infty}-\frac{1}{2} \int_{0}^{\infty} d t \int_{B}\left(p^{\infty} \partial f-f \partial p^{\infty}\right) \\
& +\frac{1}{2} \int_{0}^{\infty} d t \int_{B} p^{\infty}(\partial-k) f \\
= & \int_{0}^{\infty} d t \int_{D} f\left(-\frac{\partial}{\partial t}+\frac{\Delta}{2}\right) p^{\infty}+\frac{1}{2} \int_{0}^{\infty} d t \int_{B} f(\partial-k) p^{\infty} .
\end{aligned}
$$

By a small extension of Weyl's lemma, this formal identity implies that $p^{\infty}$ is actually of class $C^{\infty}[(0, \infty) \times(D+B-C)]$ and solves $\partial p / \partial t=\Delta p / 2$ in $D$, subject to $\partial p=k p$ on $B-C$, in the conventional sense. To finish the proof, you notice that $p^{\infty}$ is symmetric in $x$ and $y$. This is because $p^{+}$is symmetric in $x$ and $y$, as you can see from the sum $p^{+}=\sum p^{\circ}$ of article 3.4 , and this feature is inherited by $p^{\infty}$, as you can see from the formula

$$
\begin{aligned}
\frac{p^{\infty}(t, x, y)}{p^{+}(t, x, y)} & =P\left[T>t \mid \mathfrak{x}^{+}(0)=x, \mathfrak{x}^{+}(t)=y\right] \\
& =E\left[e^{-\mathfrak{f}(t)} \mid \mathfrak{x}^{+}(0)=x, \mathfrak{c}^{+}(t)=y\right] .
\end{aligned}
$$

The point is that the " $x y$ " process with sample paths $\mathfrak{x}^{+}(\mathrm{s}): s \leq t$ and probabilities conditioned by $\mathfrak{c}^{+}(0)=x$ and $\mathfrak{x}^{+}(t)=y$ is the same as the " $y x$ " process run backwards and $\mathfrak{f}(t)$ is unchanged by the substitution $\mathfrak{x}^{+}(s) \rightarrow \mathfrak{x}^{+}(t-s)$ for $s \leq t$. This symmetry permits you to conclude that $p^{\infty}$ is of class $C^{\infty}\left[(0, \infty) \times(D+B-C)^{2}\right]$. The fact that it is the only Green's function of the stated kind is now proved much as in article 3.6.

4.4 Sticky Barrier. The sticky Brownian motion associated with $\partial p=j \Delta p / 2$ is just as easy to describe. The sample paths may be expressed as $\mathfrak{x}^{\bullet}=\mathfrak{c}^{+}\left(\mathfrak{j}^{-1}\right)$ in which the "clock" $\mathfrak{j}^{-1}$ is the function inverse 
to $j(t)=t+\int_{0}^{t} j\left(\mathfrak{c}^{+}\right) d|t|$. The proof is similar to that of article 4.3. The only substantial change is that now

$$
\begin{aligned}
0 & =E \int d f\left(t, \mathfrak{c}^{\bullet}\right) \\
& =E \int_{0}^{\infty}\left(\frac{\partial}{\partial t}+\frac{\Delta}{2}\right) f\left(t, \mathfrak{c}^{\bullet}\right) d t+E \int_{0}^{\infty}\left(\partial-j \frac{\Delta}{2}\right) f(t, \mathfrak{\bullet}) d|t| \\
& =\int_{0}^{\infty} d t \int_{D} p^{\bullet}\left(\frac{\partial}{\partial t}+\frac{\Delta}{2}\right) f+\frac{1}{2} \int_{0}^{\infty} d t \int_{B} p^{\bullet}\left(\partial-j \frac{\Delta}{2}\right) f .
\end{aligned}
$$

The meaning of the adjective "sticky" is clarified if you notice that the clock $\mathfrak{j}^{-1}$ runs slower than the standard clock $t$ while $\mathfrak{r}^{\bullet}$ is on $B$, so that $\mathfrak{x} \cdot$ spends too much time on $B$, appearing to hesitate a moment before it rebounds: in fact,

$$
\text { measure }(s \leq t: \mathfrak{x} \bullet(s) \in B)=\int_{0}^{\mathfrak{j}^{-1}} j\left(\mathfrak{x}^{+}\right) d|\mathrm{t}|,
$$

as the reader will easily check. The reader may also wish to check out the case $D+B=[0, \infty)$ by hand in the style of article 4.2.

4.5 General Barries. The Brownian motion $\mathfrak{r}^{*}$ associated with $\partial p=k p+j \Delta p / 2$ is now made by running the elastic Brownian motion $\mathfrak{x}^{\infty}$ with the clock $\mathfrak{i}^{-1}$ :

$$
\mathfrak{x}^{*}= \begin{cases}\mathfrak{x}^{+}\left(\mathfrak{j}^{-1}\right) & \text { if } t<T^{*} \\ \infty & \text { if } t \geq T^{*}\end{cases}
$$

the killing time $T^{*}=\mathrm{j}(T)$ being distributed according to the conditional law $P\left[T^{*}>t \mid \mathfrak{x}^{+}\right]=\exp \left[-\mathfrak{H}\left(\mathfrak{j}^{-1}\right)\right]$. The identification proceeds much as before.

\section{BIBLIOGRAPHY}

[ 1 ] Bers, L., F. John, and M. Schechter, Partial Differential Equations, Interscience, New York, 1964.

[2] Fukushima, M., Boundary conditions for multi-dimensional Brownian motion with symmetric resolvent densities, J. Math. Soc. Japan, 21, 58-93 (1969).

[ 3 ] Itô, K., and H. P. McKean, Brownian motion on a half-line, Illinois J. Math., 7, 181-231 (1963).

[ 4 ] - Diffusion Processes and their Sample Paths, J. Springer, Berlin, 1965.

[5] Lévy, P., Processus Stochastiques et Mouvement Brownien, Gauthier-Villars, Paris, 1948. 
[6 ] McKean, H. P., Stochastic Integrals, Academic Press, New York, 1969.

[ 7 ] Sommerfeld, A., Partial Differential Equations in Physics, Academic Press, New York, 1949.

Universita di Roma, Roma, Italy

Courant Institute of Mathematical Sciences

New York University, New York, U.S.A. 